\title{
UN HABITAT ORNÉ EN ABRI SOUS-ROCHE AU MAGDALENIEN MOYEN, ANGLES-SUR-L'ANGLIN (VIENNE, FRANCE)
}

\author{
UN HÁBITAT DECORADO DEL MAGDALENIENSE \\ MEDIO EN EL ABRIGO DE ANGLES-SUR-L'ANGLIN \\ (VIENNE, FRANCIA)
}

LUDMILA IAKOVLEVA $(*)$
GENEVIEVE PINÇON $(* *)$

\section{RÉSUMÉ}

L'originalité du site du Roc-aux-Sorciers à Angles-surl'Anglin dans la Vienne (France) se traduit par la présence d'un art pariétal magdalénien sculpté dont une partie, disposée sur la paroi du fond de l'abri sous roche, forme une frise sur près de 18 mètres de long.

L'étude, tant thématique que technique ou stylistique, de cet art pariétal in situ démontre une organisation et une construction dans ce complexe figuratif sculpté.

Les sujets, aussi bien dans leur traitement que dans leur rendu, répondent à des règles précises propres au site d'Angles. Ils se regroupent par espèce en s'associant à d'autres sujets animaliers ou humains dans des espaces nettement délimités formant ainsi une composition sculptée. Des zones figuratives rassemblant plusieurs de ces compositions sculptées assimilées à des "panneaux", sont mises en relation avec le positionnement de grands foyers installés sur la roche-mère.

Cette analyse nous amène à mettre en valeur une structure complexe d' habitat en liaison avec cette frise pariétale.

\section{RESUMEN}

La originalidad del yacimiento de Roc-aux-Sorciers en Angles-sur-l'Anglin, en Vienne (Franci), se debe a la exis-

(*) Docteur en Préhistoire, Chercheur de l'Institut d'Archéologie de l'Académie Nationale des Sciences d'Ukraine, Kiev, 252014 rue 40 Vidubetskaia.

(**) Chercheur, chargée de mission au Musée des Antiquités Nationales, BP 3078103 Saint-Germain-en-Laye, France. E-mail: pinson@culture.fr

L'article a été remis dans sa version finale le 9 octobre 1998. tencia de un arte parietal magdaleniense esculpido del que una parte, situada en la pared del fondo del abrigo, forma un friso de $18 \mathrm{~m}$. de largo, aproximadamente.

El estudio, tanto temático como técnico o estilístico, de este arte parietal in situ demuestra una organización en este complejo figurativo esculpido.

Las figuras, tanto en su tratamiento como en su expresión, responden a reglas precisas, propias del yacimiento de Angles. Se agrupan por especies, asociándose a otras figuras animales o humanas, en unos espacios estrictamente limitados, formando así una composición esculpida. Las zonas figurativas que integran muchas de estas composiciones, asimiladas a " paneles ", se ponen en relación con la ubicación de unos grandes hogares instalados sobre la rocamadre.

Este análisis nos lleva definir una estructura compleja de hábitat asociada con este friso parietal.

Mots clés: Art pariétal. Habitat. Magdalénien. Sculpture. Roc-aux-Sorciers. Angles-sur-l'Anglin.

Palabras claves: Arte parietal. Hábitat. Magdaleniense. Escultura. Roc-aux-Sorciers. Angles-sur-l'Anglin.

Parmi les principaux gisements du Magdalénien moyen de France, le site d'Angles-sur-l'Anglin tient une place fondamentale. C'est un important gisement, s'étendant sur près de cinquante mètres, de la région du Poitou dans le centre de la France. Cet abri-sous-roche s'ouvre à une vingtaine de mètres au dessus de la rivière, dans une falaise calcaire 


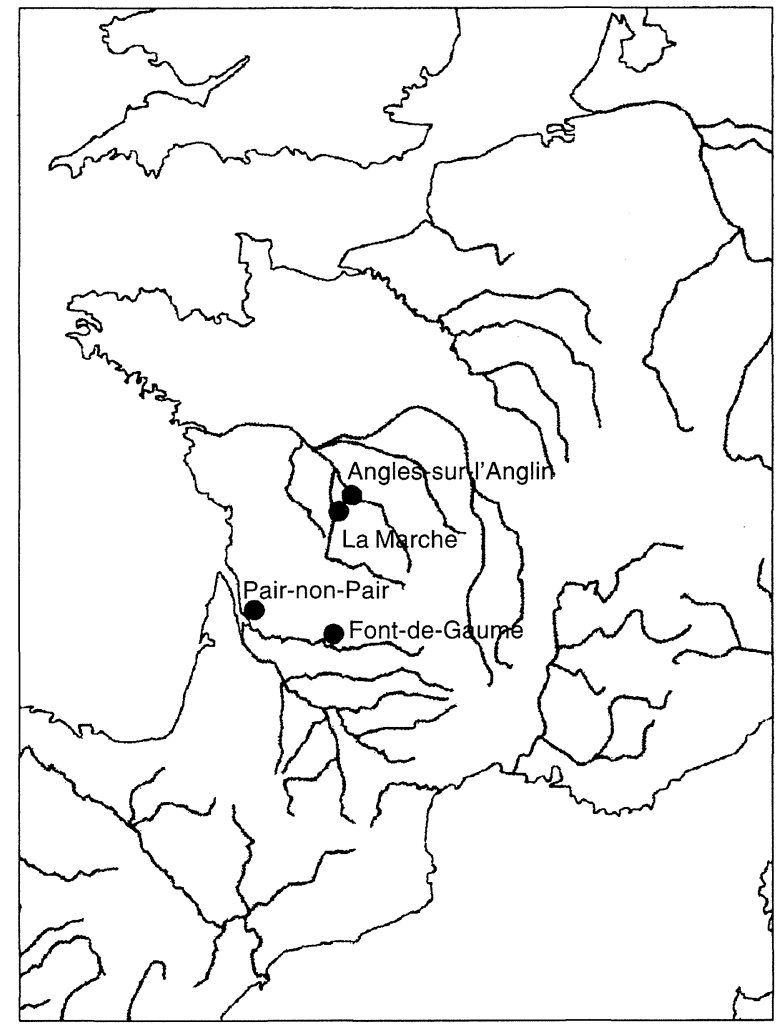

Fig. 1. Localisation des sites mentionnés.

du Néo-jurassique. On y distingue une structure d'habitat complexe associée à une frise sculptée tout au long de la paroi longeant la rivière (Pl. I).

Les Magdaléniens se sont installés sur la roche mère et ont laissé de nombreuses traces de leurs diverses activités en ces lieux. A sein des ces vestiges utilitaires ou esthétiques comme les industries variées en silex et en os, ou encore l'art mobilier et la parure, concentrés autour des grands foyers, on repère un ensemble de pièces qui témoigne des manifestations artistiques qui ont changé et se sont développées autour de cette époque à Angles, vers $14.510 \pm 160$ ans B.P. (GifA 94191) et $14.770 \pm 140$ ans B.P. (GifA 94190) pour l'occupation principale.

L'originalité de ce gisement est évoquée par l'existence d'une grande frise sculptée restée en place dans une partie du site sur une longueur de dix-huit mètres. A cet endroit, l'abri formant une niche, assez longue et peu profonde, a été mis à profit par l'artiste pour construire son œuvre. C'est précisément dans cette partie du site, moins touchée par les perturbations géologiques, que de grands foyers ont pu être repérés le long de la paroi sculptée.

Malheureusement, l'autre partie du gisement plus en amont, formant une cave, a subi un fort effondrement et seuls quelques vestiges de sculpture et une image de bison sont restés in situ sur la paroi. Plusieurs blocs tombés, trouvés dans le remplissage de cette partie du gisement, laissent penser que l'ensemble sculpté s'étendait sur tout le gisement comme une unité artistique créée pendant le Magdalénien.

La frise restée en place est constituée de plusieurs compositions sculptées animalières ou humaines qui associent toutes les techniques de l'art paléolithique (gravure, sculpture et peinture). Elle a été exécutée au cours du temps à plusieurs reprises.

Nous avons constaté qu'il y avait eu un grand travail de préparation de la paroi afin d'obtenir un support de qualité pour sculpter. Il apparaît clairement que le support a été exploité pour localiser les œuvres, en s'appuyant sur la ligne horizontale formée par la voûte. En plus de ce repère horizontal, les artistes ont partagé la paroi en différentes zones qui sont toutes bien délimitées par des lignes verticales anguleuses. Dans chacun de ces espaces aux deux bords verticaux en relief angulaire, une composition sculptée a été créée que nous avons assimilée à un "panneau" -terme courant utilisé dans les descriptions de l'art paléolithique.

On peut penser que ces lignes verticales servirent de repères pour rythmer l'œuvre. Ainsi, la frise présente une succession de panneaux. Mais malgré tout, une unité se dégage du fait de l'inscription des sculptures dans un registre principal et continu aligné sous la ligne de voûte horizontale.

Dans ce grand ensemble figuratif, nous obser-

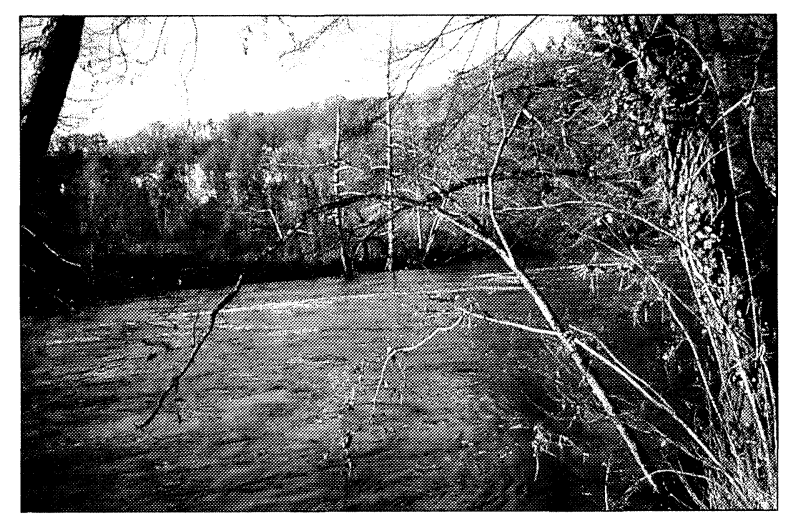

Pl. I. Le gisement du Roc-aux-Sorciers à Angles-surl'Anglin (Vienne, France): paysage (photo G. Pinçon) 


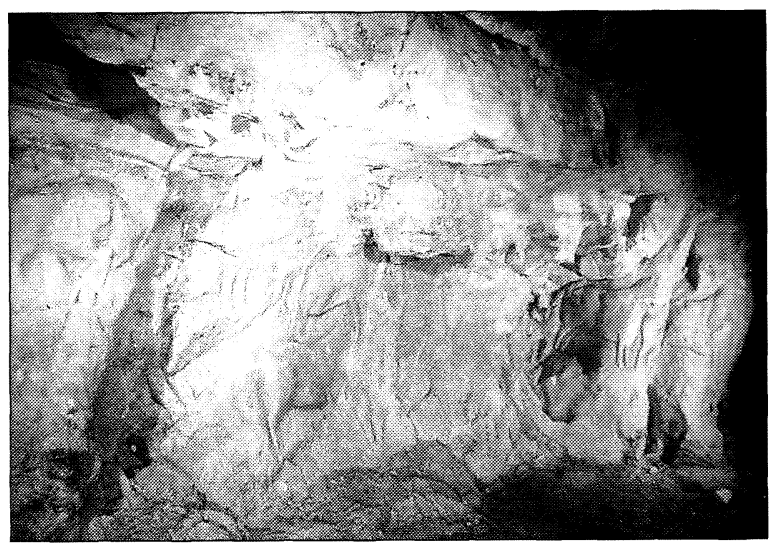

Pl. II. Vue générale de la frise sculptée (photo L. Iakovleva et G. Pinçon).

vons un placement groupé des figures animalières de même espèce (Pl. II). Nous distinguons ainsi les panneaux des bisons, les panneaux des chevaux et les panneaux des bouquetins. Ce positionnement touche également les représentations humaines, en particulier les femmes.

Au sein de certains panneaux, on observe également l'association de sujets comme des équidés à un lion, ou des femmes à deux bisons, ou encore des bouquetins à une femme.

La particularité des sculptures se marque dans l'utilisation de reproduction des sujets à des échelles variées comme la grandeur nature qui a été gardée pour les représentations féminines et les bouquetins, la moitié ou le quart de la grandeur nature utilisés pour les reproductions d'autres espèces animalières comme les bisons, les chevaux et les félins. La variété des réductions est associée à différents types de reliefs. A Angles, les volumes sont rendus à l'aide d'une grande variété de reliefs passant du relief léger, au bas-relief aux bords plus ou moins abrupts jusqu'au haut relief modelé.

Le relief fait saillie sur le fond auquel il adhère. Il correspond à une sculpture qui donne un certain volume au sujet figuré. Selon l'épaisseur voulue, les artistes ont marqué plus ou moins une zone de dégagement qui correspond à la matière enlevée lors de l'exécution de la sculpture.

Tout d'abord, nous notons comme relief léger, un relief à la fois gravé et légèrement modèle. Dans ce cas, on n'observe pas vraiment de zone de dégagement, comme par exemple pour les équidés et le lion qui leur est associé. Le bas-relief concernant la majorité des figures, comme les chevaux ou les bisons, représente moins de la moitié du volume réel d'un corps. La zone de dégagement est dans ce cas plus marquée. Dans les sculptures d'Angles, on remarque deux types de bas-relief, au bord abrupt et au bord arrondi. Un autre type de relief repéré à Angles est le demi-relief. Il s'agit d'une sculpture dont le volume en saillie correspond à la moitié du volume réel d'un corps. Une des Vénus en est un remarquable exemple. Les artistes ont également largement utilisé le haut-relief, avec une zone de dégagement large et profonde, qui présente le volume du corps avec un modelé donnant un effet proche du volume réel. Ce type de relief se retrouve pour plusieurs bouquetins.

La série de gros pics et de burins massifs retrouvés dans l'industrie lithique de ce gisement ont probablement servi aux sculpteurs pour réaliser leurs oeuvres (Saint-Mathurin, 1984: 584).

A Angles, on observe une chaîne opératoire dans la réalisation des sculptures magdaléniennes qui pour la plupart ont des contours achevés. Les artistes choisissent l'emplacement des figures en utilisant la morphologie de la paroi. En particulier, fréquemment, un bord d'arête naturelle est utilisé pour placer la tête d'un animal ce qui aide à la mise en évidence d'un volume. Le placement de différentes espèces suit ce modèle tout en respectant le cadre fixé par les arêtes naturelles et la limite de la voûte. Dans plusieurs compositions, les sujets sont placés très proches les uns des autres sans espace vide entre eux ce qui donne une unité au panneau. Cela se retrouve pour les quatre Vénus alignées, les bouquetins ou encore le couple de bisons. Dans certains cas, l'artiste a préparé plus largement le champ avec soin comme, par exemple, pour les deux bouquetins, se suivant, les plus en amont (Pl. III). Ceci renforce également l'unité de ce panneau.

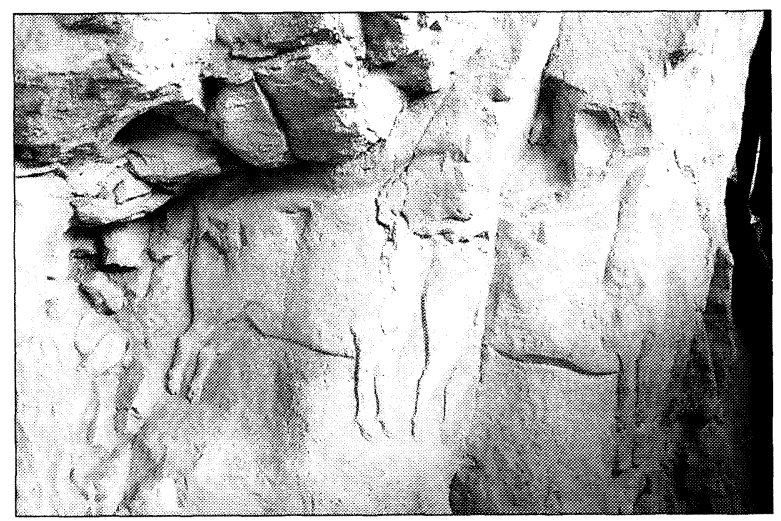

Pl. III. Les deux bouquetins en amont (photo L. Iakovleva et G. Pinçon). 
Les artistes accentuent le contour des sujets en gravant profondément les détails anatomiques et en même temps, ils polissent toute la surface des figures. Ils achèvent ces oeuvres par la peinture en deux couleurs préférentielles, le rouge et le noir. Malheureusement, aujourd'hui, cette dernière étape n'apparaît que sous la forme de vestiges.

L'utilisation de plusieurs variantes de technique de sculpture a permis aux artistes de mettre en évidence le réalisme de ces images. Tout en gardant une échelle respectant les proportions de la nature, ils jouent sur les différents reliefs et modelés pour rendre tel ou tel caractère propre au sujet qu'ils veulent figurer. Assez fréquemment, les artistes ont volontairement accentué le sexe et quelquefois l'âge des sujets, mais ont également animé ces images.

Parmi les différents thèmes animaliers, les représentations de bouquetins sont majeures dans la frise sculptée restée en place. L'ensemble des bouquetins, sculptés grandeur nature, se situe dans une zone délimitée vers l'aval par un plan de fracture d'une faille, et vers l' amont par un retour de la paroi important. Nous avons observé huit images de bouquetins qui montrent chacune une représentation du réalisme accentué par différentes variations dans les détails. Le réalisme de représentation des bouquetins traduit par les artistes d'Angles a permis le rapprochement de ces figures avec le genre $\mathrm{Ca}$ pra et plus particulièremnet l'espèce Capra Ibex ou Bouquetin des Alpes.

Parmi la série des bouquetins, on peut repérer un groupe majeur qui rassemble des bouquetins adultes mâles (Pl. II et IV). Selon la position respective de cinq d'entre eux sous la voûte, on peut voir une file de bouquetins mâles dans laquelle se distinguent deux bouquetins se suivant, un bouquetin tête inclinée et deux bouquetins affrontés. Chacun de ces sujets représente une image particulière du bouquetin. Leur représentation grandeur nature et leurs détails anatomiques soulignés par l'artiste permettent de préciser le caractère de chacun. Le premier des deux sujets se suivant montre par plusieurs traits le caractère proprement maculin de cette espèce. $\mathrm{La}$ puissance de ce mâle robuste bien proportionné par rapport au Capra Ibex (Couturier, 1962) est rendue par le volume en haut-relief modelé surtout au niveau du thorax et du poitrail tournés vers l'observateur et par la position solidement appuyée sur le sol de ses quatre pattes. Dans cette position, l'artiste présente le sabot dans tous ses détails, bien tendu, dirigé vers le sol. Le caractère masculin de ce sujet est aussi traduit par les détails de sa tête. Celle-ci a été retrouvée fracturée contre la paroi au moment des fouilles et a pu ainsi être raccordée, en particulier grâce à l'empreinte laissée au niveau du museau. La barbiche portée par les mâles Capra se distingue nettement sur cette partie de la tête.

Le sujet mâle suivant est figuré dans le même profil et présente un modèle corporel proche du premier. Il garde aussi des proportions proches de la nature. Le naturalisme de sa robustesse et de sa massivité est mis en valeur par son poitrail également sculpté en haut-relief modelé et ses pattes robustes et trapues. Ces deux mâles montrent par les détails anatomiques de la tête, tels que les bases des cornes bien développées et la barbiche, leurs caractères masculins. En même temps, par leur oeil ouvert, les oreilles dressées, la bouche ouverte, ils montrent leur vitalité. Celle-ci est encore accentuée par la position des pattes appuyées sur le sol.

Le troisième mâle sculpté plus à gauche après une zone de faille sous la voûte, en gardant la même ligne visuelle que les deux précédents, est figuré dans le même profil. Il offre une image particulière qui se traduit par sa tête inclinée, sa langue tirée, son poitrail épais. Son caractère mâle est indiqué par la représentation du sexe, de la barbiche et de ses cornes bien développées. Cet animal sculpté en basrelief montre une différence par rapport aux deux précédents par son exécution en bas-relief qui affecte tout son corps. Mais il garde en même temps des traits ressemblants avec les deux sujets précédents qui se traduisent par leurs arrière-trains en bas-relief pour les trois sujets. Une différence également peut être mise en évidence dans la représentation des détails de la tête. En effet, l'originalité de ce sujet peut s'expliquer par le remplacement d'une ancienne image de bison par ce bouquetin. Sous la voûte, la zone de dégagement du contour de la ligne cervico-dorsale encore visible rappelle la ligne dorsale d'un bison, détruit pour réaliser l'image de bouquetin. Cela explique la déformation de ce bouquetin. Il est intéressant de préciser qu' au cours du changement de thème, on peut observer sur deux sujets différents, ce bouquetin et un bison sur bloc retrouvé dans l'autre partie du gisement plus en amont, la même façon de figurer une tête de profil avec l'indication d'une langue tirée. En effet, dans ces deux cas, la tête est sculptée en relief, la langue est indiquée en relief léger en arrière-plan donnant un effet de perspective.

Encore plus à gauche, placés également sous la voûte, suivant toujours la même ligne horizontale, après un plan de faille verticale, deux mâles s'af-

T. P., 56, n. ${ }^{\circ} 1,1999$ 
frontent en regardant chacun vers l'aval dans un sens opposé à celui du regard des trois précédents bouquetins.

Ces deux bouquetins sont sculptés aussi en hautrelief au niveau du thorax et du poitrail et en basrelief pour le reste, tout en respectant les proportions du sujet grandeur nature.

Le mâle, sculpté en profil gauche, est une image de bouquetin mâle adulte, avec l'indication de la barbiche et du sexe, ainsi que des cornes bien développées et très courbes sur l'une desquelles est encore visible une nodosité.

Le contour de cet animal est impressionnant par sa massivité que l'artiste a accentuée par une ligne très convexe qui montre le large développement du poitrail et du ventre ainsi qu'une ligne de dos très creuse donnant également de l'ampleur au sujet.

En créant plusieurs niveaux de volumes, le créateur a, dans la même continuité, fait ressortir l'épaule et renforcé encore sa musculature comme pendant la marche. L'artiste a conjugué l'ensemble des moyens pour mettre en valeur la bête vivante, masculine et puissante. Il appuie sur le corps plusieurs lignes liées à la musculature, les traitant avec force par des raclages importants. Ceci souligne l'image du mâle robuste. Nous observons pour ce seul animal une technique originale en sculpture que l'on retrouve dans les peintures et gravures paléolithiques.

Affronté à ce bouquetin, l'artiste a sculpté un autre bouquetin mâle. Il présente une silhouette très ramassée sur lui-même avec un poitrail volumineux en position retournée, sur ses pattes trapues et robustes. La pose de cet animal est statique par rapport au mouvement ample donné au précédent.

Cette figure montre l'importance de la forte liaison entre la morphologie de la paroi et la représentation de ces images. Dans ce cas, nous voyons une composition placée au bord de la roche saillante, le long d'une faille. L' arrière-train de ce bouquetin est parfaitement calé sur le bord de la paroi saillante et donne la possibilité d'y lire un épisode de vie du bouquetin telle qu'on peut l'observer dans la nature quand le bouquetin, pendant ses déplacements, est en position difficile sur un relief abrupt et qu'il regarde vers le vide.

La tête d'un bouquetin en bas-relief de profil gauche a été sculptée en bordure de faille. Elle a été retaillée partiellement. L'artiste, en retaillant le volume du chanfrein d'un ancien bison, en a exagéré la concavité pour mettre en valeur un trait remarquable du bouquetin. Son caractère mâle est rendu par le grand développement de ses cornes et sa bar-

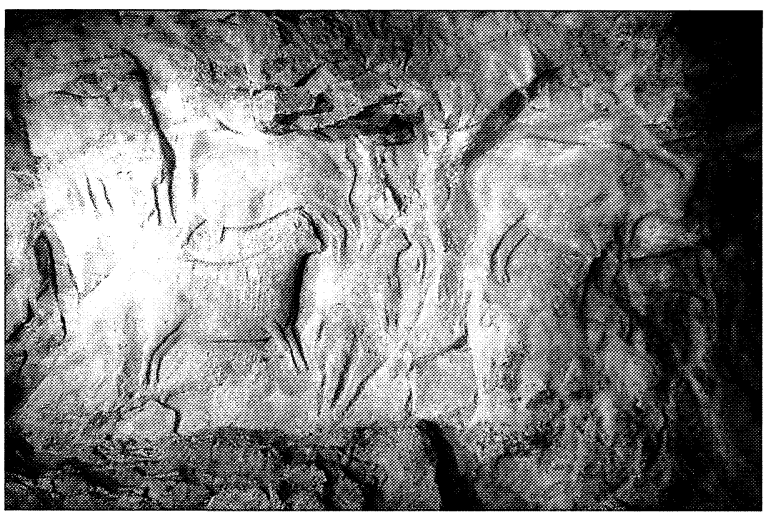

Pl. IV. Vue générale des panneaux des bouquetins (photo L. Iakovleva et G. Pinçon).

biche. Cette image a été détruite partiellement pour la mise en place d'une jeune bouquetin femelle, ce qui, encore une fois, met en évidence le changement du thème au cours du temps dans cette partie de la frise.

Cette jeune femelle est également figurée grandeur nature en respectant les proportions de l'espèce. Son corps est assez massif et épais, mais en même temps avec des pattes graciles par rapport aux pattes des mâles (Pl. IV). L'absence de barbiche et la convexité de sa ligne de ventre montrent le caractère proprement femelle de ce sujet ainsi que ses cornes rectilignes courtes, proches par leurs mensurations de celles d'une jeune femelle. Quand on mesure la longueur de la corne et la flèche, c'està-dire sa courbure, cet animal se rapproche d'une Capra Ibex actuelle d'un à deux ans (Couturier, 1962). Ces observations ne font aucun doute sur le sexe et la jeunesse de l'animal.

Malgré tous les caractères propres à ce sujet, des traits stylistiques le rapprochent des autres bouquetins de la frise, comme sa silhouette d' animal complet avec la tête en profil et le poitrail légèrement retourné. L'épaisseur du poitrail rendu par le modelé du volume de cette partie du corps, est simulée de la même façon que pour les mâles mais avec beaucoup moins d'ampleur.

On observe un traitement soigneux des pattes avec la difficulté de la forme à peine brisée du coude pour mettre en valeur son allure bondissante. Ceci est accentué par le traitement des pattes avec les sabots aux onglons et boulets bien achevés. De plus, la perspective est rendue et donne encore plus de vitalité à sa course.

Pour souligner la jeunesse de son sujet, l'artiste a rendu l'allure du jeune bouquetin bondissant avec 
les pattes jointes et tendues, la queue dressée, les yeux bien ouverts et les oreilles dressées. Ces détails donnent toute sa vitalité à ce jeune sujet femelle.

Cette jeune femelle veille sur le cabri dont les proportions correspondent à un sujet jeune. Il est présenté de profil droit, les pattes jointes et tendues. L'artiste l'a sculpté en relief léger pour signifier un très jeune animal. Sa vitalité est exprimée par le détail du museau et sa queue en position dressée.

Le sculpteur a joué sur le volume pour obtenir une perspective et a placé le petit bouquetin dans une zone concave pour le faire ressortir dans un second plan frontal, plus profond, en opposition avec la forme convexe où est figurée la "Bouquetine" qui ressort sur le premier plan frontal. Il s'agit donc, par cette opposition d'une mise en perspective, de deux images de tailles différentes mais de même profil et se suivant. L'artiste utilise différentes perspectives parfaitement adaptées aux images et au support de la paroi.

Cette association de deux jeunes bouquetins traduit une unité dans un groupe qui comprend, de plus, une petite tête de capridé en relief léger, située elle aussi sur un autre plan plus lointain.

Dans cette partie de la frise rassemblant un grand ensemble de bouquetins, on peut observer les différents sexes et âges qui correspondent à la nature. Sous la voûte, on a pu décrire cinq mâles complets auxquels s'ajoute un mâle dont seule la tête est restée. Par ailleurs, un petit groupe de deux jeunes animaux se suivant comprend un sujet femelle et un très jeune sujet. Ce placement volontaire de cinq mâles sur le même registre par rapport au petit groupe de jeunes bouquetins dans un autre registre, peut avoir une signification particulière où l'on peut lire une dualité sexuelle séparée.

Dans cette zone, le changement de thème se traduit par la destruction de certaines sculptures remplacées par d'autres. Cela s'observe dans la position du bouquetin mâle tirant la langue qui est placé dans une ancienne silhouette de bison. Par ailleurs, la destruction partielle de figure est lisible dans la superposition du cabri avec un corps nu féminin, vu de face, qui a été préservé partiellement juste entre les pattes du cabri. Cette image de femme partielle a été exécutée dans une technique différente de celle des autres femmes figurées dans la frise. En effet, on observe le piquetage qui a permis son dégagement grossier. Le bord est resté brut de façonnage sans aucun modelage, ce qui est bien différent des autres figures de femme. Cette différence est en relation avec les proportions plus courtes du corps, les hanches épaisses et les jambes plus courtes. Dans ce cas, la figure féminine présente un thème plus ancien que celui des bouquetins qui eux-mêmes ont remplacé partiellement les bisons qui sont détruits dans cette partie de la frise. Dans cette zone figurative, on observe le changement du thème au cours du temps, mais également l'utilisation partielle d'anciennes images s'intégrant dans les nouvelles compositions pouvant être interprétées dans un certain contexte significatif (jeune bouquetin femelle-cabri-femme) comme un thème féminin.

Dans cette partie de la frise, plusieurs traces de coloration apparaissent, en particulier la couleur noire des sabots des bouquetins mâles et les traces de couleur rouge associée à plusieurs sujets et en particulier à la jeune femelle et au cabri. Ceci nous laisse penser qu'à l'origine les sculptures étaient enrichies d'au moins deux couleurs principales, le rouge et le noir.

Au voisinage de ce grand ensemble de bouquetins', après une zone de faille importante, plus en aval, se retrouve le groupe féminin associé à deux bisons qui montre la préservation de ces sujets qui, ailleurs, ont été remplacés par les bouquetins comme nous l'avons vu précédemment.

Le groupe féminin présente, outre les vestiges (nombril, ligne de dos) d'un corps féminin, trois femmes sveltes, sculptées grandeur nature, placées côte à côte et mises en place selon une ligne horizontale (Fig. 2).

Chacune montre une image bien individuelle par sa position et ses détails figurés. Leur réalisme est rendu volontairement par l'artiste, mais en même temps, ces Vénus présentent une particularité de création par l'absence des membres supérieurs, l'absence de l'extrémité des membres inférieurs et l'absence de tête. En même temps, on observe l'association d'uneVénus avec une tête sculptée (Fig. 2 ,b), positionnée de façon légèrement décalée par rapport à ce corps mais peut être en composition avec le corps féminin et ayant une certaine signification.

Ces femmes présentent des caractères communs propres au site d'Angles en particulier au niveau des techniques, de la composition et des proportions par rapport aux autres images de femmes de cette époque qui nous sont connues dans plusieurs gisements.

Le contour du tronc des femmes d'Angles est fait de la même façon pour chacune, en dégageant beaucoup de matière et en laissant un espace triangulaire qui met en relief de part et d'autre chacun des troncs des figures. La zone de la cuisse et du fessi- 


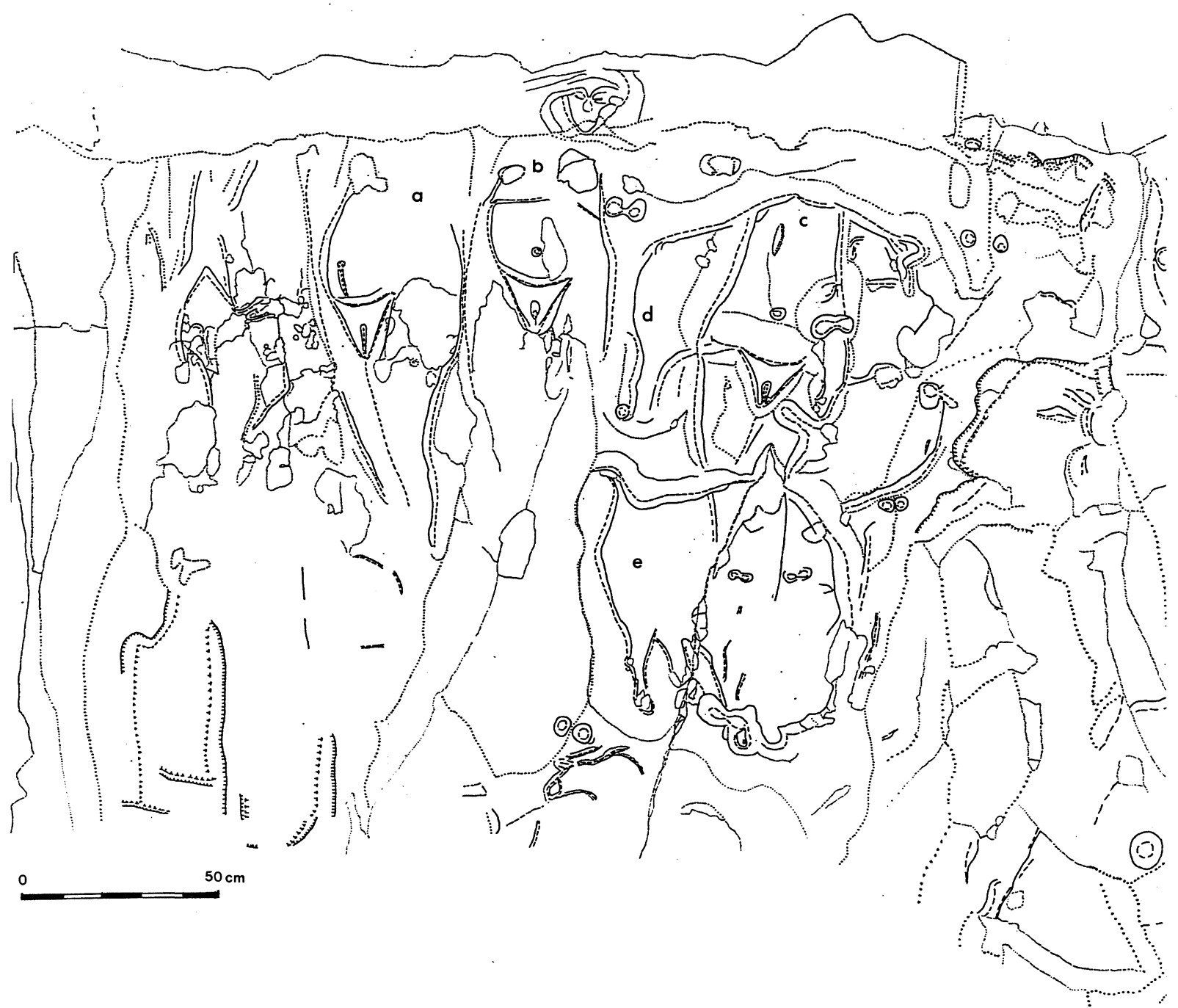

Fig. 2. Relevé du panneau des Vénus (relevé de L. Iakovleva et G. Pinçon, 1996).

er est plane par rapport au ventre et marquée par les lignes dessinant les jambes. Le ventre avec son nombril est accentué par son relief.

La particularité de ces figures est dans l'absence des membres et de la tête mais surtout dans l'accent volontairement mis par l'artiste pour le traitement du triangle pubien de chacune qui est sculpté avec soin. Celui-ci est profondément gravé. Il est façonné à partir d'incisions très profondes, linéaires, régulières, en $\mathrm{V}$ symétrique, disposées symétriquement par rapport à la vulve. Nous constatons là encore les bords arrondis de la gravure. Le sexe féminin est encore plus accentué par le côté fini de la gravure profonde. Tout en gardant la même dimension pour le triangle pubien, on peut voir une différence pour chacune dans la représentation de la vulve. L'une est façonnée par une simple incision et un très léger volume. Pour une autre, la vulve, réalisée à partir d'un concavité naturelle, est large et ouverte. Pour la suivante, elle marque un volume léger.

Ainsi, ces figurations féminines, présentées côte à côte à même hauteur, ont les mêmes proportions, mais en même temps, elles offrent chacune leur propres caractères individuels dans leur nudité.

Le réalisme, lors de la création de ces sujets est clairement traduit par les mensurations et les proportions qui correspondent à la réalité.

Dans ce groupe de Vénus, on peut observer la particularité de chacune par sa position, l'une présentée de trois-quarts (Fig. 2,a) par rapport aux autres qui sont de face (Fig. 2,b,c). De plus elles 
portent des détails différents reflétant la morphologie du corps féminin bien individualisé (Iakovleva et Pinçon, 1995: 123-128).

L'individualisme du corps féminin peut être interprété très largement. On peut observer de grandes variations entre les femmes, selon leur âge, leur morphologie individuelle, leur pathologie et leurs différents états de gravidité. Ceci est révélé par la forme et le volume des seins comme aussi par le volume et la forme de leur ventre et de leurs hanches.

A Angles, le groupe des femmes peut avoir différentes interprétations. Au sein de ce groupe, laVénus sculptée en demi-relief avec le ventre bombé (Fig. 2 ,b) a été interprétée comme la représentation d'une femme gravide et parturiente (Saint-Mathurin et Garrod, 1951: 417-419; Duhard, 1993: 197) et la Vénus à sa gauche comme une multipare. LaVénus superposée aux bisons a été interprétée comme une femme venant d'accoucher (Duhard, 1993: 197).

Mais selon toutes les interprétations variées possibles, il faut remarquer l'individualisme du corps féminin que l'on peut interpréter assez différemment. Par exemple, la Vénus associée aux bisons est une représentation d'une femme avec la poitrine plate sans les seins, le ventre plat et et les hanches sans graisse. On peut y voir l'image d'un corps svelte de jeune femme. Cela nous semblerait plus logique par rapport à une image de femme après accouchement, qui, d'après la réalité, garde pendant un certain temps un ventre plus volumineux et surtout les seins gonflés pendant la période d'allaitement.

De même, on peut faire une interprétation différente de laVénus vue de trois-quarts. Nous sommes devant une femme qui, d'après sa position, permet de bien lire le grand ventre bombé avec une ligne marquée entre le nombril et le triangle publien qui peut signifier la ligne sombre liée à la pigmentaton de certaines femmes brunes pendant leur grossesse. Il est tout à fait possible que l'image de cette femme reproduise une femme enceinte. Ceci n'exclut pas l'hypothèse d'une femme multipare qui aurait un certain âge avec un ventre volumineux et les hanches plus épaisses et de ce fait déformées.

Avec toutes ces remarques, nous gardons l'image de ce groupe de femmes comme une forme de représentation de la nature avec toutes ses variétés que les Magdaléniens ont pu observer dans leur société.

En même temps, ce panneau complexe présente un sens artistique significatif particulier donné par les deux images de bisons qui se superposent au groupe de femmes.
A Angles, on retrouve une femme associée à deux bisons sculptés en bas-relief dans une taille réduite par rapport à la grandeur nature des Vénus. L'un est figé, l'autre animé est superposé partiellement aux pieds de cette femme.

Ces deux bisons de même profil droit, juxtaposés sur un axe vertical, sont reproduits en bas-relief aux bords abrupts, comme la Vénus à laquelle ils sont superposés. Ils sont tous les deux dans les mêmes proportions réduites, proches du quart de la grandeur nature.

Le bison figé (Fig. 2,d) est sculpté juste sous la voûte, la femme vient se superposer de façon centrée par rapport au corps de l'animal. Ce bison est amorphe sans aucun signe d'animation. La patte antérieure de ce bison, légèrement fléchie, marque sa position figée. Ce caractère s'accentue par le façonnage des pattes restées inachevées. Sa ligne cervico-dorsale est interrompue par la Vénus mais elle a bien une forme qui souligne les caractéristiqués du bison. Cette morphologie du bison est marquée également par le contour de l'ornement pileux du poitrail et celui de la barbe qui est légèrement de forme angulaire.

L'autre bison (Fig. 2,e) montre au contraire une attitude fortement animée traduisant un dynamisme. Dans cette situation, le genre mâle est le plus souvent figuré. La représentation du caractère sexuel primaire de cet animal donne une image du bison mâle vivant, avec des traits d'agressivité.

Ce bison a une image personnelle à cause de la forme de son support particulièrement convexe et de ses traits de stylisation. Il est délimité à droite sur un bord angulaire, et à gauche par une longue fissure verticale. Ce cadrage serré, sur un support bien délimité de la paroi particulièrement convexe, donna à l'artiste l'impulsion de créer cette image dynamique. Le corps du bison a été stylisé volontairement en ramassant le bison sur lui-même, en le resserrant entre les deux bords verticaux de la paroi. Il est représenté dans une proportion proche du quart de la grandeur nature, ce qui est en bonne concordance avec le bison situé au-desssus.

Ici, l'artiste ne se préocupe pas des rapports de proportions exacts, il joue de ces dimensions anormales pour créer une image originale par son dynamisme. L'artiste a sculpté un anneau qui brise les pattes antérieures. En surchargeant ainsi cet animal d'un anneau, il a, malgré tout, épargné des éléments anatomiques qu'il jugeait importants pour garder l'image du bison animé.

Dans cette composition, c'est-à-dire deux bisons

T. P., 56, n. ${ }^{\circ} 1,1999$ 
alignés verticalement, présentés de même profil et superposés à une femme nue vue de face, on peut lire par la position des animaux, le bison amorphe et le bison animé, la signification dans la représentation animalière, d'une dualité d'opposition, comme statisme/dynamisme et en même temps, en allant plus loin dans l'interprétation, mort/vie.

La Vénus qui se superpose à ce thème complique cette composition.

Ce groupe de deux bisons (figé-animé) superposés à une femme a probablement un sens particulier à Angles. Il faut bien remarquer que, dans cette représentation, le bison animé est situé aux pieds de la Vénus. La position dynamique d'un des bisons peut traduire de l'agressivité, une offensive, ou une attaque, face à laVénus qui est en position inoffensive. Cette attitude a été systématiquement reproduite pour toutes les représentations féminines à Angles.

Grâce à ces représentations, nous pouvons penser que les artistes ont figuré ces sujets en mettant en évidence l'opposition et en même temps le rapprochement entre les humains et les animaux, du fait de leur mode de vie réciproque à l'époque paléolithique. Les artistes ont utilisé leur talent pour rendre l'attitude sereine féminine en oppositon à l' attitude plus offensive masculine traduite par le bison mâle.

Cette composition complexe met en évidence le dualisme sexuel bien représenté dans l'art paléolithique (Laming-Emperaire, 1962; Leroi-Gourhan, 1965).

A Angles, le thème du bison est largement présent par ailleurs. La représentation la plus proche du panneau des Vénus se situe en aval, juste audessus de la niche avec les deux chevaux (Pl.V). Ce bison reproduit proche de la moitié de la grandeur nature est figuré en bas-relief, de profil gauche, dans une position couchée, pattes repliées. Il regarde vers l'aval où se situe un autre panneau présentant un couple de bisons. Ceux-ci sont à la même échelle, également en bas-relief, mais debout de profil droit. Ces deux bisons se suivant, sculptés en position statique, présentent un mâle à la tête inclinée et une femelle le suivant, fracturée anciennement. La particularité de cette composition sculptée est mise en évidence par la sexualité notée de ces deux animaux (Iakovleva et Pinçon, 1996).

L'attention des artistes d'Angles pour les bisons est mise en évidence par les autres images de cette espèce retrouvées dans les fouilles de l'autre partie en amont du gisement. Seule une belle image est restée in situ sur la voûte de la cave.

Dans cette partie du gisement, parmi les blocs

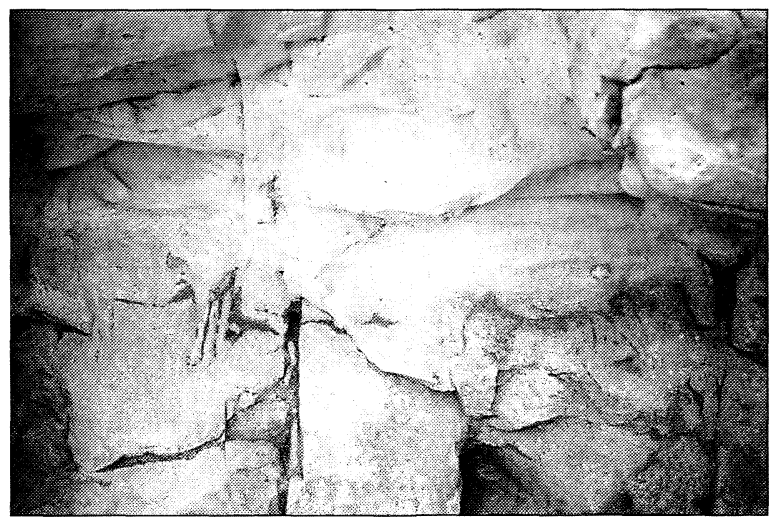

Pl. V. Les chevaux et le bison couché (photo L. Iakovleva et G. Pinçon).

tombés, plusieurs représentations de chevaux ont été mises au jour. Parmi celles-ci, on peut remarquer, en particulier, quatre chevaux sculptés, malheureusement fracturés, trouvés proches les uns des autres (Saint-Mathurin, 1988: 4,5 et 4,9). Ils montrent malgré tout, chacun, un réalisme traduit par leur taille proche de la grandeur nature, leur volume sculpté en bas et haut-relief modelé, et leur profil identique. Par leur position, ils peuvent être interprétés comme une partie de frise effondrée. Ces images mettent en évidence la représentation de chevaux groupés. Dans l'autre partie du gisement, cette espèce est également figurée.

Plusieurs de ces chevaux sont proches du cheval sauvage de Prjewalski par leur morphologie. Cela est illustré par deux images proches de la moitié de la grandeur nature, sculptées de profil en bas-relief dans le registre principal de la frise (Pl. V).

L'artiste présente une image de cheval de profil gauche, la tête tournée à droite, proche du modèle vivant tout en apportant des caractères personnels comme la tête tournée dans des proportions artificielles. Le réalisme de la figure est rendu par la ligne de l'encolure interrompue qui suggère le prolongement du mouvement invisible pour l'observateur que nous sommes.

Toutes les extrémités sont achevées et soulignent la finesse de création de la silhouette de cet animal. Nous sommes devant un cheval au repos, en position statique, queue tombante, debout sur ses pattes figées graciles.

L'originalité de cette représentation animalière a obligé le sculpteur à caler l'image complète de l'animal juste sous la voûte. Le support de paroi a donc dicté à l'artiste cette disproportion de la tête, mais il montre tout son talent en traitant l'animal 
dans cette position particulière pour obtenir un sujet complet. La position de la tête a été volontairement choisie. La tête est tournée vers le fond de la paroi. De même, le corps est plus court pour accentuer son volume, plus important à cause du mouvement qui affecte aussi le poitrail. L'artiste a essayé de sculpter en utilisant volontairement les disproportions pour montrer l'animal se retournant.

Cette disproportion est encore accentuée par la technique. En effet, le corps est en volume, particulièrement convexe, ce qui appuie encore l'aspect volumineux de ce cheval. Du fait de la forme de la paroi, l'artiste a été obligé de façonner la tête en dégageant beaucoup de matière afin de la faire ressortir de la surface, à cet endroit un peu en retrait justement. La tête et le corps ne sont pas sur le même plan. La tête n'apparaît plus dans sa totalité pour ce qui est de sa plus grande longueur comme dans un profil absolu. Etant plus éloignée de l'observateur que le corps, elle apparaît plus petite. Cette création donne une image de perspective étudiée. L'animal tourne la tête, celle-ci est plus éloignée de l'observateur que le corps. Cette figuration en relief est accentuée par l'ombre donnée par la niche qui donne un effet particulier et esthétique.

Ce trait d'animation de l'animal qui tourne la tête est assez rare dans l'art préhistorique. On le retrouve à une époque plus ancienne, au Gravettien, dans la grotte de Pair Non Pair en Gironde (Delluc, 1991: fig. 57 et 58).

L'autre image de cheval sculptée juste à proximité dans le même registre, présente une silhouette en profil droit avec la tête penchée, la ligne de ventre marquée par sa convexité, et ses extrêmités bien achevées. L'exécution des pattes grêles ainsi que la tête figurée avec tous ses détails antomiques achèvent finement cette représentation de cheval. Cette image est une création originale par ses détails qui sont très nettement réalisés et proches de l'anatomie de cette espèce: grande narine, oeil bien ouvert, bouche entrouverte. Il s'agit d'une image vivante. A ceci s'ajoute le mouvement des pattes antérieures, projetées vers l'avant qui peut encore marquer un trait d'animation.

Une autre image de cheval appartient au groupe d'équidés sculptés en masse en relief léger dans une taille plus réduite. Cette composition est placée dans le registre inférieur, juste sous le grand bouquetin mâle tirant la langue. On peut y voir un regroupement de chevaux traités dans un même profil sur deux plans différents, avec deux sujets figurés partiellement et un autre sujet figuré complet. Ce mode de représentation est illustré par plusieurs exemples gravés sur des plaquettes de la grotte de la Marche (Vienne), géographiquement proche d'Angles.

Nous avons une image de cheval vivant que l'artiste a traduit par la représentation de la tête penchée avec la figuration de tous les détails précisés avec finesse, ainsi que les pattes au contour achevé et détaillé avec, en particulier, la couronne bien notée. La position de la patte avec la tête baissée jusqu'à la ligne de sol, nous invite à voir un animal en train de boire ou de brouter. Cette même attitude se retrouve dans deux autres images sur blocs, citées plus haut.

Cette composition de chevaux met en évidence un autre type d'association animalière figurée à Angles, qui est la superposition d'un lion à ce groupe d'équidés (Pl. IV). Dans cette composition, on peut lire une symétrie dans la disposition spatiale des animaux qui se répartissent entre le carnivore et les herbivores. Le lion est figuré en relief léger, en taille réduite, mais contrairement aux équidés, il est de profil droit.

Dans cette composition, les deux sujets sont peu détaillés (il s'agit des deux équidés présents en deuxième plan) et les autres ont les détails anatomiques traités avec soin (il s'agit du petit cheval broutant et du lion).

Les deux sujets, le cheval broutant et le lion, ont des traits d'animation qui se traduisent pour l'un par sa tête penchée, pour l'autre par sa tête tendue.

Dans cette composition complexe, on peut voir un certain symbolisme traduit par l'association d'herbivores avec un carnivore, c'est-à-dire les animaux chassés et les animaux chasseurs. Dans cette association significative, on peut lire une réalité de la nature associant chasseur-herbivores où dans le premier plan est figuré le chasseur et dans l'arrière plan, le troupeau d'équidés.

En même temps, il faut noter que cette composition ne représente pas une scène de vraie chasse dans laquelle plus logiquement les animaux seraient dans le même profil ou affrontés et non dos à dos comme c'est le cas ici. Dans la composition d'Angles, peut être observée une originalité artistique du traitement de ce thème.

L'existence de cette association particulière peut être illustrée par une autre composition dans l'art magdalénien. A Font-de-Gaume (Dordogne) un félin est gravé face à des chevaux. Dans la complexité de ce thème, le félin, en profil complet, est associé à des chevaux complets ou partiels, figurés de différentes façons. 
La présence d'un autre félin, sculpté partiellement, dans le même registre inférieur doit être remarquée. Cette tête de félin, en profil absolu, est sculptée en relief à bords abrupts faisant ressortir le danger émanent de cet animal. Ce sujet sculpté grandeur nature présente un profil droit sobre. Sont marquées la narine, la bouche ouverte et l'indication d'un oeil. Le polissage de sa surface traduit une image partielle mais achevée, adaptée au support de la paroi qui s'arrête brutalement sur une zone de faille. Cet animal dangereux à la gueule ouverte vient s'opposer à un bouquetin à l' attitude paisible qui est placé sur une même verticale naturelle.

On peut voir ces images sculptées partiellement, ne représentant que des têtes, comme deux entités traduisant le danger et la sérénité. La position symétrique sur un même axe, face à face, de ces figures accentue cette dualité. La position d'un côté de la tête de félin de profil droit, et de l'autre côté la tête de capridé de profil gauche créées dans une même dimension, se situent sur un même niveau. Ces deux images sont placées de chaque côté d'une faille qui porte une composition géométrique en peinture rouge. D'après leur position particulière, ces deux espèces, carnivores et herbivores, ressortent bien sur la paroi.

Dans l'association félin-chevaux, les sujets sont de taille plus réduite et sont comparables dans la représentation des détails donnant les traits d'animation de chacune des espèces, en particulier du félin. Il est étonnant de retrouver ces deux félins aussi proches sur la paroi, avec chacun des caractères marquant leurs différences. L'un est présenté partiel, grandeur nature, mettant en évidence son caractère dangeureux avec sa gueule ouverte, sans l'accentuation d'autres détails. L'autre est figuré complet, de plus petite taille mais avec des caractères anatomiques plus soulignés. Il apparaît le cou tendu vers l'avant et sa tête porte des détails appuyant sa vivacité comme sa bouche ouverte, sa narine respirant et l'oeil qui regarde, ce qui met en évidence la vitalité de cet animal.

Il est intéressant de souligner un trait particulier à Angles pour cette espèce. En effet, contrairement aux animaux chassés comme les chevaux, les bisons ou les bouquetins, le félin est toujours représenté isolé de façon complète ou partielle. Ce mode de représentation est repérable tout au long du site, y compris sur un bloc de la partie amont du gisement qui porte une image sculptée en relief léger de lion, partiel mais de grande taille, vu de profil droit.
Le groupement ou l'isolement des sujets animaliers peut traduire la différence du comportement des espèces dans la nature, repéré et volontairement retenu par les artistes magdaléniens. Le mode de représentation différent des sujets animaliers groupés comme les herbivores ou au contraire du sujet isolé notamment le carnivore, peut être vu comme un trait caractéristique dans la tradition artistique d'Angles.

Dans la représentation humaine, on peut observer également une différence entre la représentation féminine en groupe par rapport à la figuration de la tête humaine isolée. Celle-ci est présente plusieurs fois tout au long du gisement. Une tête sculptée en demi relief, vue de face, a été placée légèrement décalée au-dessus d'un corps féminin. Par sa taille, elle est proportionnelle au corps de la femme. Une autre tête humaine, sculptée en relief léger, est ausssi vue de face. Elle est placée juste sous le bouquetin mâle tirant la langue. Elle évoque plutôt un simple masque. En revanche, une troisième tête humaine est figurée de profil droit, également en relief léger sous un autre bouquetin plus en amont. Cette image, un peu exagérée, présente un profil souriant. Son caractère propre est traduit par son nez long et droit, sa bouche large et son menton anguleux. Des traces de couleur noire visibles sur le visage laissent à penser qu'à l'origine ce profil était enrichi en couleur.

Une autre tête humaine qui est connue (SaintMathurin, 1976) sur un bloc effondré, offre un tout autre caractère qui lui est propre. Il s'agit d'une tête d'homme sculptée en bas-relief, de profil droit. Ce profil est nettement sculpté en bas-relief au bord abrupt, mettant en évidence le caractère singulier de ce personnage au front bombé et au nez retroussé. Cette partie de la tête porte les traits caractéristiques de la physionomie de ce personnage avec le large nez épaté et épais, avec la notation en gravure profonde de larges narines. Du fait d'un endommagement ancien du bout du nez et de la bouche, on peut juste noter l'extrémité des lèvres gravées. Le profil de cette tête est achevé par une ligne large et profonde qui souligne le menton. Une autre ligne gravée parallèlement indique une barbe courte. $\mathrm{La}$ peinture noire vient s'ajouter pour fortement accentuer l'espace des cheveux et de la barbe. Il est intéressant de souligner que cet homme regarde. Sous le sourcil légèrement courbe gravé, l'oeil est figuré en amande, avec l'indication de la caroncule lacrymale et celle de la pupille, marquée par un point de couleur noire et entourée de cils. 
Toutes ces observations montrent que les artistes ont traduit certaines images des Magdaléniens en prenant en compte la particularité de chacun de façon certainement exagérée, mais en gardant la tradition générale d'Angles de rendre le réalisme dans les oeuvres.

D'après tout ce que nous venons de présenter sur la frise restée en place, on distinguent le regroupement d'animaux de même espèce dans différents espaces. Dans certaines zones, des sujets animaliers de même espèce sont aussi associés à d'autres figures animalières ou humaines.

Nous avons ainsi observé différentes zones figuratives dans lesquelles se distingue un ou plusieurs panneaux. Les zones figuratives sont séparées entre elles par des zone de failles non utilisables pour réaliser des sculptures du fait de la morphologie de la paroi. Dans chacune des zones figuratives, on observe le placement des compositions sculptées -"panneaux"- selon des règles propres à Angles. Chaque panneau a été délimité entre deux reliefs angulaires naturels qui portent des anneaux sculptés alignés sur la verticale. Dans le registre principal, la majorité des panneaux ont été alignés naturellement sous la ligne de voûte. Dans certains cas, des reliefs angulaires horizontaux sont également chargés d'anneaux.

Suivant cette construction, nous comptons, d'aval en amont, huit panneaux figuratifs : un couple de bisons, un cheval tournant la tête, un bison couché, un cheval marchant, un ensemble de Vénus associées à deux bisons, un ensemble de bouquetins, un bouquetin mâle associé à des équidés et un félin, deux bouquetins. Plusieurs zones figuratives sont en relation avec le positionnement de grands foyers installés sur la roche-mère. Cette association se comprend du fait de la morphologie de l'abri plus ou moins en retrait.

D'après cette organisation, on peut noter différentes parties distinctes dans cet habitat sous roche. Chacun de ces espaces restreints a pu être protégé en utilisant des anneaux comme éléments de structure d'habitations. Celles-ci ont pu être différemment fonctionnelles au cours des occupations au Magdalénien moyen (Iakovleva et Pinçon, 1997).

Pour conclure, on peut retenir, à Angles, l'idée de la succession de petits habitats tout au long de la frise. Dans le cas d'une fermeture, ces espaces ainsi délimités étaient éclairés par des foyers placés sur la roche mère juste au pied de ces panneaux. La frise sculptée pouvait être alors visible à l'aide d'un éclairage artificiel de façon segmentaire.
L'originalité de cet habitat se reflète, en particulier sur la paroi, à la lecture des différents panneaux offrant chacun distinctement leur propre composition. Cette organisation thématique n'empêchait pas les Magdaléniens de connaître la frise dans sa continuité.

Aujourd'hui, la mise à la lumière du jour de cet immense ensemble sculpté le fait apparaître comme une unité visuelle, et reste pour nous un témoin important des manifestations artistiques développées à cette époque.

\section{BIBLIOGRAPHIE}

COUTURIer, M. (1962): Le bouquetin des Alpes. Imp.Allier. Grenoble.

Delluc, B. et G. (1991): "L'Art pariétal archaïque en Aquitaine”. XXVIIIe suppl. à Gallia-Préhistoire. C.N.R.S. Paris.

DuHARD J.-P. (1993): Réalisme de l'image féminine paléolithique. Ed. C.N.R.S. Cahier du Quaternaire, 19.

Iakovleva, L. et Pinçon, G. (1995): "Les représentations féminines dans l'art pariétal du Roc-aux-Sorciers à Angles-sur-1'Anglin (Vienne). Colloque international sur l'image de la femme dans l'art paléolithique. Brassempouy, Juillet 1994. Ed. Etudes et Recherches Archéologiques de l'Université de Liège. ERAUL, 74: 123-128.

- (1996): "Une Composition de deux bisons sculptés de la frise de l'abri Bourdois àAngles-sur-l' Anglin (Vienne)". BSPF, 93(2): 195-200.

- (1997): La Frise sculptée du Roc-aux-Sorciers à Angles-sur-l'Anglin (Vienne). Ed. R.M.N. et C.T.H.S. Paris.

Laming-Emperaire A. (1962): La Signification de l'art rupestre paléolithique. Picard. Paris.

Leroi-Gourhan, A. (1965): Préhistoire de l'art occidental. Mazenod. Paris.

SAINT-MATHuRIN DE, S. (1976): "Reliefs magdaléniens d'Angles-sur-l'Anglin (Vienne)". Antiquités Nationales, 7: 24-31.

- (1984): "L'Abri du Roc-aux-Sorciers". L'Art des Cavernes. Atlas des grottes ornées paléolithiques françaises. Ministère de la Culture. Impr. Nat. Paris: 283-287.

- (1988): "Les Sculptures rupestres du Roc-aux-Sorciers". L'art préhistorique, 1: Les grands sites de Poitou-Charentes, Dossier Histoire et Archéologie. Archeologia, 131: 42-49.

Saint-Mathurin DE, S.et Garrod, D.A.E. (1951) : "Nouvelles découvertes dans l'abri du Roc-aux-Sorciers àAngles-sur-l'Anglin (Vienne): 'les Vénus paléolithiques"'. Comptes-rendus de l'Académie des Inscriptions et Belles-Lettres. Séance du 9 février: 51-57. 